\title{
THE TRANSFER OF ANTIBIOTIC RESISTANCE BETWEEN STRAINS OF ENTEROBACTERIA IN CHICKEN, CALVES AND PIGS
}

\author{
H. WiLliams Smith \\ Animal Health Trust, Stock, Essex
}

Plate XI

WHEN antibiotic resistance is determined by the presence of an $\mathrm{R}$ factor, its transfer from one enterobacterial strain to another can usually be demonstrated without difficulty in vitro, even when it occurs at a low rate. From the practical point of view, it is important to know the extent to which it occurs in vivo.

The available evidence indicates that transfer is much more difficult to demonstrate in vivo than in vitro (Watanabe, 1963). Apart from resort to the selective use of antibiotics, its demonstration in vivo has usually been achieved by first modifying the normal flora of the alimentary tract. Kasuya (1964) and Guinée (1965), for example, demonstrated it in mice whose normal intestinal flora had been reduced by the preliminary feeding of antibiotics; Kasuya, and Salzman and Klemm (1968) also obtained positive results in germ-free mice. Walton (1966) approached the problem by administering R-factor-bearing strains of Escherichia coli, by mouth, to chicken immediately they had hatched and their alimentary tracts were sterile. Prospective recipient strains were given $6 \mathrm{hr}$ later, before food and water were made available. By this technique, antibiotic resistance was transferred from one strain of $E$. coli to another and from E. coli to Salmonella typhimurium.

The present paper is concerned with the transfer of antibiotic resistance in chicken with a normal alimentary flora; in some experiments with these animals $\mathrm{R}$ factors spread in epidemic fashion from $E$. coli to $S$. typhimurium. Less extensive studies with calves and pigs are also reported.

\section{MATERIALS AND METHODS \\ Antibiotic-sensitivity tests}

Approximately $0.1 \mathrm{ml}$ of a 1 in 100 dilution of a $24-\mathrm{hr}$ broth culture of the bacterial strain to be tested was spread evenly over the surface of a dried petri plate containing Disk Sensitivity Test (DST) agar (Oxoid, CM261) and an Oxoid Multodisk (1744E) was applied. The Multodisk was composed of 8 disks containing (i) streptomycin (S) $25 \mu \mathrm{g}$, (ii) ampicillin (A) $25 \mu \mathrm{g}$, (iii) oxytetracycline (T) $50 \mu \mathrm{g}$, (iv) chloramphenicol (C) $50 \mu \mathrm{g}$, (v) neomycin (N) $30 \mu \mathrm{g}$, (vi) nalidixic acid (X) $30 \mu \mathrm{g}$, (vii) furazolidone (F) $15 \mu \mathrm{g}$, and (viii) sulphonamide (Su) $300 \mu \mathrm{g}$.

The plates were incubated at $37^{\circ} \mathrm{C}$ for $24 \mathrm{hr}$ and read. No difficulty was experienced in making readings; the zones of bacterial inhibition surrounding the disks were either wide, very narrow or absent.

\section{Transfer of antibiotic resistance in vitro}

Nutrient broth (Oxoid no. 2) in 10-ml amounts was seeded with $0.02 \mathrm{ml}$ of a 24 -hr broth culture of the prospective donor strain and $0.02 \mathrm{ml}$ of a similar culture of the prospective recipient strain. Nalidixic acid-resistant mutants of antibiotic-sensitive strains were always used as prospective recipient strains unless the donor itself was also a nalidixic acid-resistant

Received 29 Apr. 1969; accepted 26 July 1969.

J. MED. MICROBIOL.-VOL, 3 (1970) 
mutant; in that case a spectinomycin-resistant recipient strain was used. All the resistant mutants, except those of $E$. coli $\mathrm{K} 12$, were smooth. When only the transmissibility of the resistance of a strain was being tested, an $E$. coli ${\mathrm{K} 12 \mathrm{~F}^{-}}^{-}$strain was used as the prospective recipient. The mixed culture of prospective donor and recipient strains was incubated at $37^{\circ} \mathrm{C}$ in a waterbath for $24 \mathrm{hr}$ and then inoculated, sometimes as centrifugate, on a plate of MacConkey medium containing nalidixic acid and one of the antibiotics to which the prospective donor was resistant and the prospective recipient was sensitive. The plate (referred to as selection medium) was incubated at $37^{\circ} \mathrm{C}$ for $24 \mathrm{hr}$. If any colonies of the recipient strain grew upon it, one or more were purified by replating and their drug sensitivity pattern determined. The concentrations of antibiotic used in the selection medium were $20 \mu \mathrm{g}$ per $\mathrm{ml}$ for nalidixic acid, oxytetracycline, chloramphenicol, neomycin, spectinomycin and ampicillin and $15 \mu \mathrm{g}$ per $\mathrm{ml}$ in the case of streptomycin.

In some cases the proportion of organisms of the prospective recipient strain in the mixed culture that had acquired antibiotic resistance was estimated. This was done by performing viable counts according to the method of Miles and Misra (Miles, Misra and Irwin, 1938) on MacConkey agar containing nalidixic acid alone and with one or other of the antibiotics to which the donor strain was resistant.

\section{Transfer of antibiotic resistance in vivo}

All the prospective recipient strains of $E$. coli and salmonellae used in the in-vivo transfer studies were smooth nalidixic acid-resistant mutants, organisms that are rare in animal communities. This, together with the fact that they were easily identifiable by slide agglutination tests, made it extremely unlikely that extraneous organisms entering the alimentary tract of the experimental animals would be wrongly identified as being of the recipient strain. The use of selection media containing nalidixic acid also meant that, for all practical purposes, the only organisms that would grow on this medium after it had been inoculated with faeces were those of the recipient strains. Furthermore, it seemed very unlikely that R-factor transfer would occur on the surface of the medium after seeding with faeces, because it did not occur in preliminary experiments in which plates of the medium were seeded with broth cultures of good donor and recipient strains immediately after they had been mixed.

Transfer experiments with chicken. For their first 3 days of life, male Arbor Acre sexlinked chicken, usually in groups of 17 , were supplied with drinking water to which had been added a suspension of the prospective donor strain grown on nutrient agar; the final bacterial concentration was $10^{7}-10^{8}$ viable organisms per $\mathrm{ml}$ of drinking water. A proprietary meal containing neither antibacterial nor anticoccidial drugs was provided ad libitum. On the 4th day the contaminated drinking water was replaced by clean water and each chick was given by mouth $10^{9}$ viable organisms of the prospective recipient strain grown on nutrient agar and administered as a culture suspension. At the same time, cloacal swabs were taken from about one-third of the chicken and cultured on plates of brilliant green agar (Oxoid, CM263) or MacConkey agar, containing nalidixic acid $20 \mu \mathrm{g}$ per $\mathrm{ml}$ to confirm the absence of organisms of, or resembling, the prospective recipient strain; brilliant green agar was used when the prospective recipient strain was a salmonella, and MacConkey agar when it was E. coli. The swabs were also cultured on a plate of plain MacConkey agar and a Multodisk (1744E) was applied to indicate the extent to which the prospective donor strain of $E$. coli had colonised the faeces. At this time, estimates were also made of the total faecal flora of some of the chicken by the methods of Smith (1965). The flora was similar to that found by Smith in normal 4-day-old chicken and consisted principally of $E$. coli, streptococci, lactobacilli and bacteroides.

Three days after the chicken were given the prospective recipient strain, cloacal swabs were taken and each one was smeared over the surface of a plate of culture medium containing nalidixic acid $20 \mu \mathrm{g}$ per $\mathrm{ml}$ and a Multodisk was applied; MacConkey agar was used when the prospective recipient organisms were $E$. coli and brilliant green agar when they were salmonellae. The plates were incubated for $24 \mathrm{hr}$ at $37^{\circ} \mathrm{C}$ and read, attention being paid to the amount of growth and to the zones around the disks to see whether they contained 
TRANSFER OF ANTIBIOTIC RESISTANCE

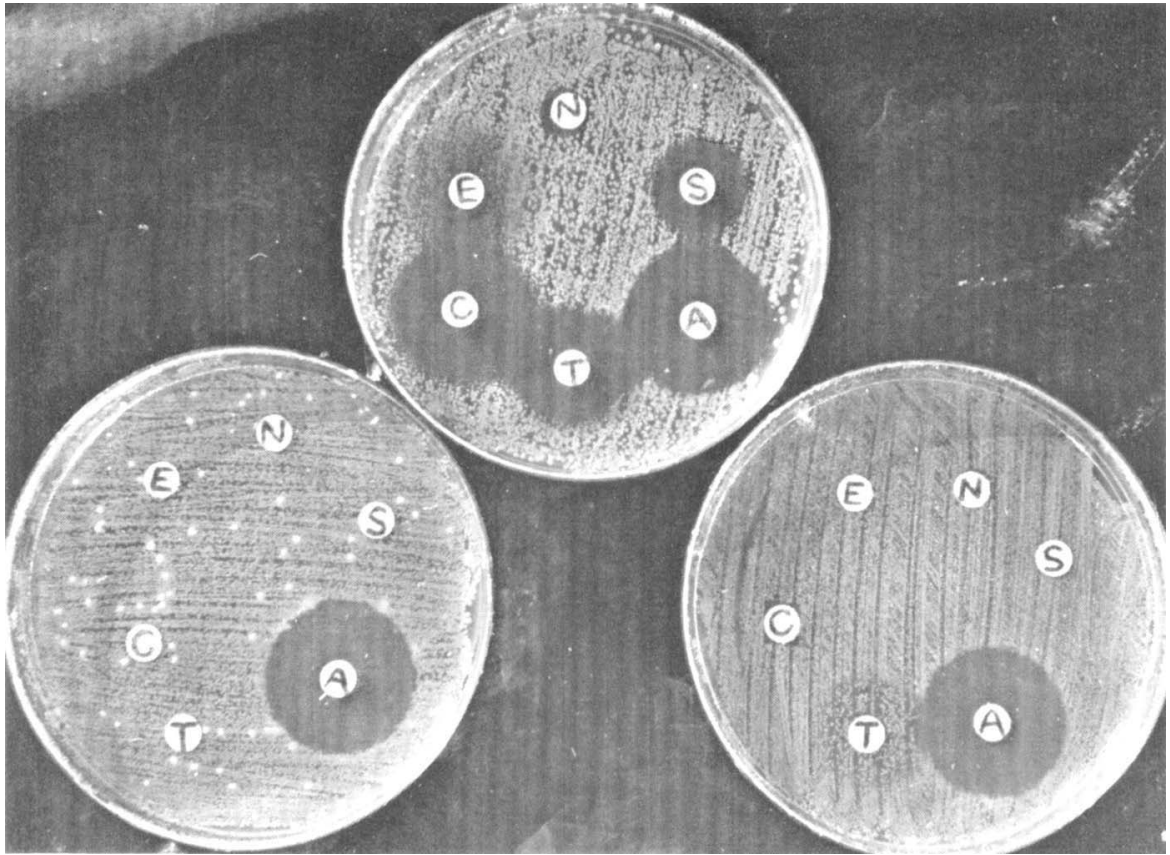

FIG. 1.-Direct sensitivity tests on the predominantly S. typhimurium content of cultures of faeces from 3 chicken given a nalidixic acid-resistant mutant of $S$. typhimurium strain 576, 3 days after they had been given $E$. coli P18 with transmissible resistance pattern SSu TC. For clarity, individual sensitivity disks were used in these tests instead of the customary Multodisk. Not all the organisms in the faecal sample inoculated on the right petri plate had acquired tetracycline resistance. $\mathrm{A}=$ Ampicillin; $\mathrm{S}=$ streptomycin; $\mathrm{T}=$ tetracycline; $\mathrm{C}=$ chloramphenicol; $\mathrm{E}=$ sulphonamide; $\mathrm{N}=$ nalidixic acid. $\quad c . \frac{2}{3}$.

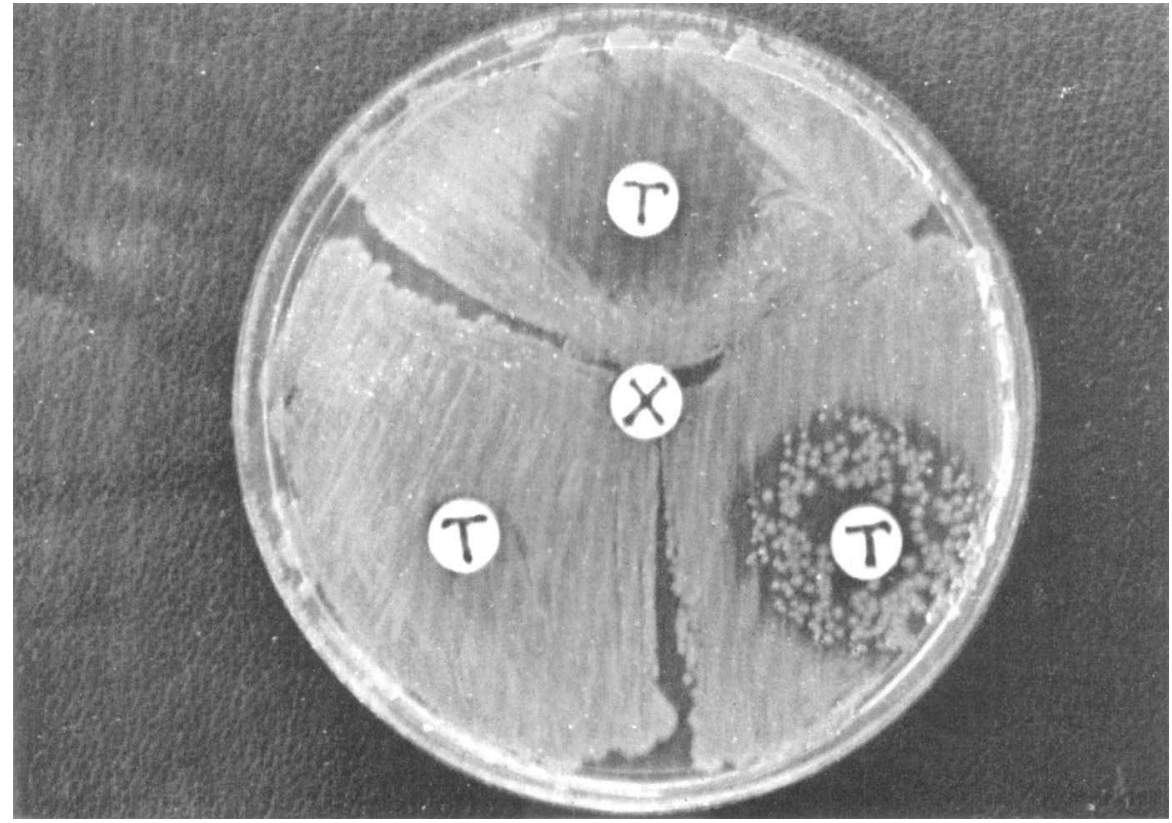

FIG. 2.- Sensitivity tests on S. typhimurium organisms subcultured from selenite broth cultures of the livers of 3 chicken given a nalidixic acid-resistant mutant of $S$. typhimuritum strain 576, 3 days after they had been given $E$. coli $\mathrm{F} 21$ with transmissible resistance pattern $\mathrm{T}$. $\mathrm{T}=$ Tetracycline; $\mathrm{X}=$ nalidixic acid. $\times c .1$. 
resistant colonies. If they did, some of them from some of the plates were submitted to slide agglutination tests to confirm that they were of the recipient strain, and their antibiotic resistance pattern was determined. The transferable nature of the resistance was also demonstrated when this was thought necessary.

Three days later the chicken were killed and their faeces were examined in the manner described above. Their livers were also examined in the same way except that the bacterial content was first enriched in selenite medium; the livers did not usually contain enough organisms to permit the satisfactory performance of direct sensitivity tests. These selenite cultures, however, could not be regarded as reliable indicators of the proportion of resistant and sensitive organisms in the livers because preliminary experiments with mixed cultures revealed that R-factor transfer between salmonella organisms could occur in selenite broth.

After completion of each experiment, the metal cages in which each group of chicken were housed and their feeding utensils were immersed in a disinfectant solution, thoroughly cleaned, re-disinfected and flame-gunned before being used again. Each cage was kept in a separate compartment of the animal house, the floors of which were disinfected between each experiment. In most experiments, 8 groups of chicken were used, each group sometimes being given a different prospective recipient strain. On no occasion was cross-infection with organisms of the prospective recipient strains detected.

Transfer experiments with calves. Male Jersey calves were brought to the laboratory from a neighbouring farm when they were less than $24 \mathrm{hr}$ old and after they had obtained colostrum. They were housed in clean pens, no more than one calf being present in the laboratory at any one time. They were fed on reconstituted dried milk, 1 litre twice daily. Immediately on arrival each calf was given $10^{10}$ viable organisms of a strain of E. coli, F18, that had been shown to be a good R-factor donor in chicken. On the next day, they were given, again by mouth, $10^{8}-10^{10}$ viable organisms of a nalidixic acid-resistant mutant strain of $S$. typhimurium suspended in $50 \mathrm{ml}$ of milk. Their faeces were examined, immediately on arrival and at daily intervals, in the same manner as the chicken faecal samples were examined. If the calves became very ill they were killed and the chyme from different parts of the alimentary tract, a mesenteric lymph-node, the spleen and liver were examined in the same manner as the faeces had been.

Transfer experiments with pigs. Large White pigs aged 10-14 wk were used. They were kept in groups of 2-4 in metal pens that were cleaned, disinfected and flame-gunned between each experiment. The details of the experiments performed in these animals are referred to later; they were confined to the transfer of tetracycline resistance amongst $E$. coli.

\section{RESULTS}

\section{The transfer of antibiotic resistance in vitro}

The degree of resistance transfer that occurred in mixed cultures of most of the strains of $E$. coli and salmonellae that were subsequently used in the in-vivo studies is illustrated in table I. The original strains of $S$. typhimurium from which the nalidixic acid-resistant prospective recipient strains were prepared had been received from Dr E. S. Anderson of the Enteric Reference Laboratory, Colindale, London, who had found that none of them possessed transfer factors for antibiotic resistance; none of the other prospective recipient strains was found, in this laboratory, to possess transfer factors. Great differences were found between some strains in their donor ability, even between strains possessing the same pattern of transmissible resistance. A further 50 epidemiologically unrelated $E$. coli strains that were tetracycline-resistant only were examined for donor ability in relation to $S$. typhimurium strain 576 . None of them was as good as $E$. coli F21 (table I) in this respect; 45 were little better or no better than F20. Great differences were also found between 
strains in their recipient ability. Some of the salmonellae, including the S. typhimurium strains no. 5235 (phage-type 29) and no. 576 (phage-type 36)

TABLE I

Resistance transfer in different mixed cultures of E. coli and salmonellae

\begin{tabular}{|c|c|c|c|c|c|c|c|}
\hline \multirow{2}{*}{$\begin{array}{l}\text { Prospective } \\
\text { donor strain } \\
\text { of } E \text {. coli }\end{array}$} & \multirow{2}{*}{$\begin{array}{l}\text { Transmissible } \\
\text { resistance } \\
\text { pattern }\end{array}$} & \multirow{2}{*}{$\begin{array}{l}\text { Prospective recipient } \\
\text { strain }\end{array}$} & \multicolumn{5}{|c|}{$\begin{array}{l}\text { Approximate incidence*, in the recipient strain } \\
\text { in the mixed culture } \dagger \text {, of organisms that had } \\
\text { acquired resistance to }\end{array}$} \\
\hline & & & $\mathrm{A} \ddagger$ & $\mathrm{S}$ & $\mathbf{N}$ & $\mathrm{T}$ & C \\
\hline $\begin{array}{l}\text { F17 } \\
\text { F18 } \\
\text { F21 } \\
\text { F16 } \\
\text { F19 } \\
\text { F22 } \\
\text { P233 } \\
\text { P18 } \\
\text { F20 } \\
\text { F14 } \\
\text { P113 } \\
\text { B92 } \\
\text { H44 }\end{array}$ & $\begin{array}{l}\text { ASNT } \\
\text { ASSuNTC } \\
\text { T } \\
\text { SNT } \\
\text { ST } \\
\text { T } \\
\text { N } \\
\text { SSuTC } \\
\text { T } \\
\text { ASSuNTC } \\
\text { SSuTC } \\
\text { SSuT } \\
\text { ASSuNTC }\end{array}$ & $\begin{array}{l}\text { S. typhimurium } 5768 \\
\text { S. typhimurium } 576 \\
\text { S. typhimurium } 576 \\
\text { S. typhimurium } 576 \\
\text { S. typhimurium } 576 \\
\text { S. typhimurium } 576 \\
\text { S. typhimurium } 576 \\
\text { S.typhimurium } 576 \\
\text { S. typhimurium } 576 \\
\text { S. typhimurium } 576 \\
\text { S. typhimurium } 576 \\
\text { S. typhimurium } 576 \\
\text { S. typhimurium } 576\end{array}$ & $\mid \begin{array}{c}10^{0} \\
8 \times 10^{-1} \\
\cdots \\
\cdots \\
\cdots \\
\cdots \\
\cdots \\
\ldots \\
5 \times 10^{-4} \\
\cdots \\
\cdots \\
10^{-6}\end{array}$ & $\left|\begin{array}{c}6 \times 10^{-1} \\
8 \times 10^{-1} \\
4 \times 10^{-1} \\
2 \times 10^{-1} \\
\ldots \\
\ldots \\
10^{-3} \\
\ldots \\
10^{-7} \\
10^{-5} \\
10^{-5} \\
10^{-7}\end{array}\right|$ & $\left|\begin{array}{c}6 \times 10^{-1} \\
8 \times 10^{-1} \\
4 \times 10^{-1} \\
\cdots \\
2 \times 10^{-3} \\
\cdots \\
10^{-7} \\
\cdots \\
\ldots \\
10^{-8}\end{array}\right|$ & $\mid \begin{array}{c}10^{-2} \\
10^{-1} \\
6 \times 10^{-1} \\
4 \times 10^{-1} \\
2 \times 10^{-1} \\
10^{-2} \\
\cdots \\
10^{-3} \\
4 \times 10^{-4} \\
5 \times 10^{-6} \\
10^{-5} \\
10^{-5} \\
10^{-8}\end{array}$ & $\begin{array}{c}10^{-1} \\
\ldots \\
\ldots \\
\cdots \\
\ldots \\
10^{-3} \\
\ldots \\
10^{-7} \\
10^{-5} \\
\ldots \\
10^{-8}\end{array}$ \\
\hline $\begin{array}{l}\text { F17 } \\
\text { F17 } \\
\text { F17 } \\
\text { F17 } \\
\text { F17 } \\
\text { F17 } \\
\text { F17 } \\
\text { F17 } \\
\text { F17 } \\
\text { F17 } \\
\text { F17 }\end{array}$ & $\begin{array}{l}\text { ASNT } \\
\text { ASNT } \\
\text { ASNT } \\
\text { ASNT } \\
\text { ASNT } \\
\text { ASNT } \\
\text { ASNT } \\
\text { ASNT } \\
\text { ASNT } \\
\text { ASNT } \\
\text { ASNT }\end{array}$ & $\begin{array}{l}\text { E. coli } \mathrm{K} 12 \mathrm{~F}^{-} \\
\text {S. typhimurium } 5235 \\
\text { S. heidelberg } \\
\text { S. anatum } \\
\text { S. typhimurium } 98 \\
\text { S. senftenberg } \\
\text { S. thompson } \\
\text { S. montevideo } \\
\text { E. coli } \mathrm{H} 1(\mathrm{O} 26: \mathrm{K} 60) \\
\text { S. typhimurium } 533 \\
\text { S. arizona }(7 \mathrm{a}, 7 \mathrm{~b}: 1,7,8)\end{array}$ & $\begin{array}{c}2 \times 10^{-1} \\
2 \times 10^{-1} \\
2 \times 10^{-1} \\
10^{-1} \\
10^{-1} \\
10^{-1} \\
6 \times 10^{-3} \\
5 \times 10^{-4} \\
3 \times 10^{-4} \\
3 \times 10^{-5} \\
5 \times 10^{-5}\end{array}$ & $\mid \begin{array}{c}2 \times 10^{-1} \\
2 \times 10^{-1} \\
10^{-1} \\
10^{-1} \\
10^{-1} \\
10^{-1} \\
3 \times 10^{-3} \\
5 \times 10^{-4} \\
3 \times 10^{-4} \\
10^{-4} \\
5 \times 10^{-5}\end{array}$ & $\left|\begin{array}{c}2 \times 10^{-1} \\
2 \times 10^{-1} \\
10^{-1} \\
10^{-1} \\
10^{-1} \\
10^{-1} \\
3 \times 10^{-3} \\
5 \times 10^{-4} \\
3 \times 10^{-4} \\
10^{-4} \\
5 \times 10^{-5}\end{array}\right|$ & $\mid$\begin{tabular}{c|}
$5 \times 10^{-2}$ \\
$2 \times 10^{-3}$ \\
$10^{-2}$ \\
$10^{-2}$ \\
$10^{-3}$ \\
$10^{-3}$ \\
$5 \times 10^{-4}$ \\
$10^{-4}$ \\
$3 \times 10^{-4}$ \\
$2 \times 10^{-5}$ \\
$2 \times 10^{-5}$
\end{tabular} & $\begin{array}{l}\cdots \\
\cdots \\
\cdots \\
\cdots \\
\cdots \\
\cdots \\
\cdots \\
\cdots \\
\cdots \\
\cdots\end{array}$ \\
\hline $\begin{array}{l}\text { F18 } \\
\text { F18 }\end{array}$ & $\begin{array}{l}\text { A S Su NTC } \\
\text { A S Su NTC }\end{array}$ & $\begin{array}{l}\text { S. typhimurium } 5235 \\
\text { E. coli } \mathrm{K}_{12 \mathrm{~F}^{-}}\end{array}$ & $\left|\begin{array}{l}9 \times 10^{-1} \\
9 \times 10^{-1}\end{array}\right|$ & $\left|\begin{array}{l}9 \times 10^{-1} \\
9 \times 10^{-1}\end{array}\right|$ & $\left|\begin{array}{l}9 \times 10^{-1} \\
9 \times 10^{-1}\end{array}\right|$ & $10^{-2}$ & $\begin{array}{l}5 \times 10^{-2} \\
8 \times 10^{-1}\end{array}$ \\
\hline
\end{tabular}

* To avoid long strings of noughts, incidences are given in exponential form; $10^{0}=1$ in 1 , i.e., 100 per cent. of organisms were resistant; $10^{-6}$ means that 1 in $1,000,000$ organisms was resistant, or 0.0001 per cent.; $4 \times 10^{-6}$ means that 4 organisms per million were resistant $(0.0004$ per cent.).

$\dagger$ The mixed culture had been incubated at $37^{\circ} \mathrm{C}$ for $24 \mathrm{hr}$.

$\ddagger \mathrm{A}=$ Ampicillin; $\mathrm{S}=$ streptomycin; $\mathrm{Su}=$ sulphonamide; $\mathrm{N}=$ neomycin; $\mathrm{T}=$ oxytetracycline; $\mathrm{C}=$ chloramphenicol.

$\S$ S. typhimurium strains are listed under Enteric Reference Laboratory serial numbers; strain 576 belonged to phage-type 36 ; strain 5235 to phage-type 29; and strains 98 and 533 to phage-type 14 .

were equal to $E$. coli $\mathrm{K} 12 \mathrm{~F}$ - in some respects. Of the two $S$. typhimurium strains of phage-type 14, no. 98 was a much better recipient than no. 533 .

\section{Antibiotic-resistance transfer in chicken}

The transfer of resistance from different strains of $E$. coli to

\section{S. typhimurium strain 576}

The results of examining the faeces and livers of groups of chicken for evidence of antibiotic resistance transfer to a nalidixic acid-resistant mutant of 


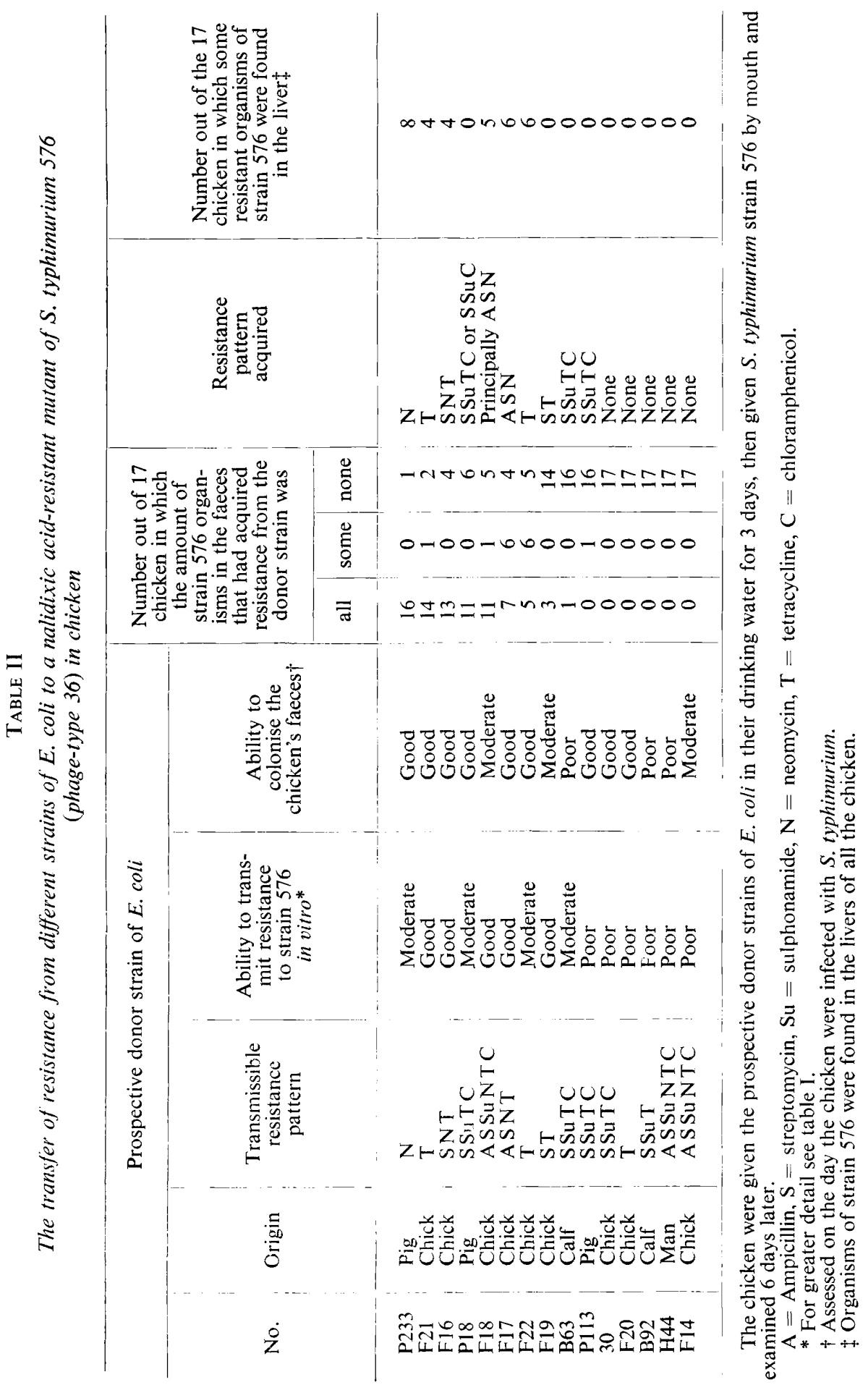


S. typhimurium 576, phage-type 36 , with which they had been infected orally 6 days previously, are summarised in table II; each group had been given a different R-factor-bearing strain of $E$. coli in its drinking water for 3 days before being given the $S$. typhimurium. Sensitivity tests on the predominantly $S$. typhimurium content of the faeces cultured from 3 of the chicken given $E$. coli P18 are illustrated in fig. 1; the tests on cultures prepared from the livers of 3 of the chicken given F21 are illustrated in fig. 2.

Resistance transfer to $S$. typhimurium 576 occurred much more commonly in some groups of chicken than in others. It was commonest in chicken that had been given strains of $E$. coli that were reasonably good donors in vitro and that were reasonably good colonisers of the alimentary tract. It was observed in only 1 of 102 chicken given strains of $E$. coli that were poor donors in vitro. Two of the good in-vivo donors, P233 and P18, had been isolated initially in pigs; P233 transmitted not only neomycin resistance in the chicken but also the ability to produce colicine.

Antibiotic-resistant $S$. typhimurium organisms were found in the livers of some of the chicken given $E$. coli P233, F16, F17, F18, F21 and F22. This indicates that in these chicken some $S$. typhimurium organisms invaded the body after receiving $\mathrm{R}$ factors from $E$. coli in the alimentary tract.

\section{The transfer of resistance from $E$. coli $F 17$ to different strains of salmonellae and E. coli}

The results of examining the faeces and livers of groups of chicken for evidence of antibiotic resistance transfer from $E$. coli F17 to nalidixic acidresistant strains of salmonellae and $E$. coli are summarised in table III. Of the strains of $E$. coli tested, $\mathrm{H} 1(\mathrm{O} 26: \mathrm{K} 60)$ in its nalidixic acid-sensitive form was responsible for neonatal diarrhoea in a human being and F1 (O1:K1) for an outbreak of bacteriaemia in broiler fowl; the $S$. arizonae strain caused outbreaks of disease in turkeys in Britain.

The incidence of antibiotic-resistant recipient organisms in the faeces of the chicken varied greatly from group to group. Such organisms commonly formed a significantly large proportion of the prospective recipient population of the faeces of those chicken given strains that had been found to be good recipients of the R factors of $E$. coli F17 in vitro (table I) and were good colonisers of the alimentary tract. This was well demonstrated by the $S$. typhimurium strains, with the exception of strain 533 , which was a poor recipient in in-vitro tests. The $S$. typhimurium strains were also more invasive than the other strains examined, and this is significantly related to the finding of resistant organisms in the livers as well as in the faeces. Little or no evidence of resistance transfer was found in those groups of chicken given prospective recipient strains such as $S$. gallinarum and $S$. montevideo that were poor recipients in vitro and poor colonisers of the alimentary tract. The faecal specimens from the 22 chicken given $S$. gallinarum and $S$. montevideo that did not yield any prospective recipient organisms when submitted to the standard test were enriched in selenite broth and retested; 10 of them then yielded positive cultures, 
TRANSFER OF ANTIBIOTIC RESISTANCE

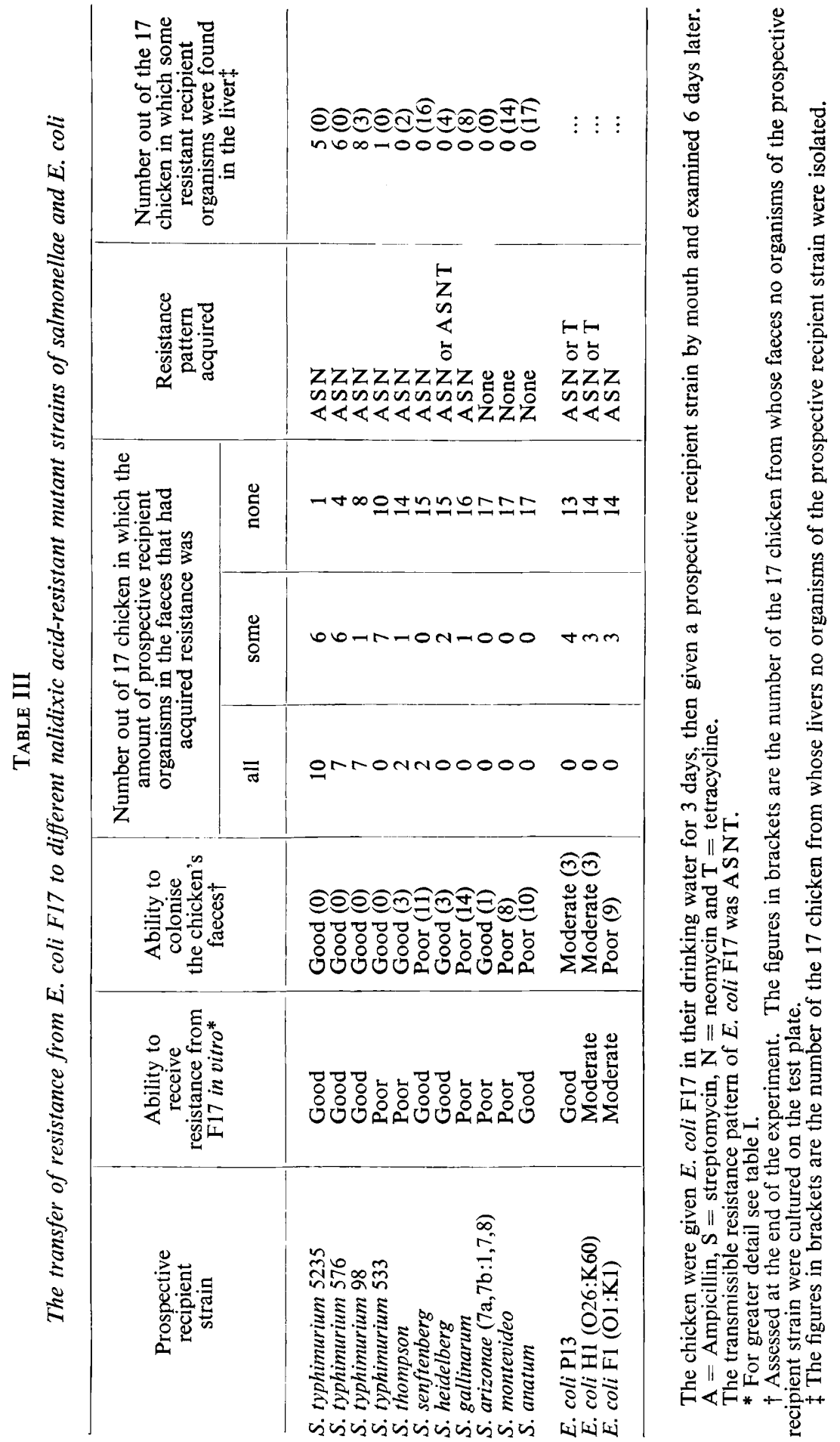


but none of these contained resistant organisms. Resistance transfer was slight or not observed in those groups of chicken given poor in-vitro recipients but good colonisers such as the test strains of $S$. thompson and $S$. arizonae, or good in-vitro recipients but poor colonisers such as the $S$. anatum and $S$. senftenberg strains. The caecal contents of the 11 chicken given $S$. senftenberg but showing no faecal carriage of the test strain, all yielded a moderate growth of $S$. senftenberg and 6 contained resistant organisms; the resistant organisms formed only a very small proportion of the total numbers of $S$. senftenberg organisms present. The low incidence of resistance transfer in

TABLE IV

The effect of time on transmission of resistance from $E$. coli to $S$. typhimurium in chicken

\begin{tabular}{c|c|c|c}
\hline $\begin{array}{c}\text { Prospective } \\
\text { donor strain } \\
\text { of } E \text {. coli }\end{array}$ & $\begin{array}{c}\text { Pumber out of } 17 \text { chicken in which all the } S \text {. typhimurium } \\
\text { Prospective recipient } \\
\text { strain of } S . \text { typhimurium }\end{array}$ & $\begin{array}{c}\text { organisms in the faeces had acquired resistance at the } \\
\text { stated time after infection with } S \text {. typhimurium }\end{array}$ \\
\hline & & 3 days & 6 days after infection \\
\hline P18 & 576 & 1 & 11 \\
F16 & 576 & 9 & 13 \\
P233 & 576 & 12 & 6 \\
F17 & 576 & 4 & 10 \\
F17 & 5235 & 5 & 10 \\
F18 & 5235 & & 17 \\
\hline
\end{tabular}

the group of chicken given $S$. heidelberg was unexpected, because this strain was both a good in-vitro recipient and a good coloniser.

The resistance pattern usually transferred in the chicken from $E$. coli F17 was resistance to ampicillin, streptomycin and neomycin (ASN), the pattern transferred most efficiently in vitro (table I); only rarely was there transfer of the full transmissible resistance pattern of strain F17 (ASNT) or resistance to tetracycline alone $(\mathrm{T})$.

\section{The effect of time on resistance transfer}

The faeces of groups of chicken were examined for evidence of resistance transfer from different prospective donor strains of $E$. coli to $S$. typhimurium strain 576 or 5235,3 and 6 days after one or other of these prospective recipients had been administered. The results (table IV) show that a greater proportion of the $S$. typhimurium population of the faeces had acquired resistance at 6 days than at 3 days.

\section{The effect of antibiotic administration on resistance transfer}

Groups of chicken were fed continuously on diets containing different antibiotics during experiments in which they were given $E$. coli F18 in their drinking water for 3 days, then infected with $S$. typhimurium 5235, phage-type 
29. Their faeces and livers were examined bacteriologically 6 days later and the results are summarised in table V. E. coli F18 possessed the resistance pattern ASSu NTCF, but only F-resistance was non-transmissible in vitro.

Except for the occasional isolation of small numbers of organisms possessing the full transmissible resistance pattern, the principal pattern possessed by the $S$. typhimurium in the faeces of the chicken given ampicillin or streptomycin or neomycin or furazolidone or no antibiotics, was ASN. This was also the principal resistance pattern possessed by the organisms isolated from the chicken given 0.15 per cent. sulphadimidine, which, at this level, was probably without influence on resistance transfer. When the test chicken were given 0.75 per cent. of this agent, the full pattern was transmitted, including resistance to Su. It is noteworthy that both the higher levels of sulphadimidine and oxytetracycline were associated with the emergence of $S$. typhimurium organisms with a resistance pattern that included resistance to chloramphenicol (C), whereas the administration of chloramphenicol, even at high levels, had no such effect. The results obtained with the chicken given chloramphenicol indeed resembled those with chicken given no antibiotics, the principal resistance pattern possessed by the resistant organisms again being A S N.

Some agents, including furazolidone and oxytetracycline and sulphadimidine at the higher levels, reduced the numbers of $S$. typhimurium organisms in the faeces of the chicken to which they were given. The reduction produced by 0.75 per cent. sulphadimidine, was so marked that no $S$. typhimurium organisms were present on the sensitivity-test plates spread with samples of faeces of 29 of the 34 chicken in this test series. This accounts for the record in table $\mathrm{V}$ that some chicken had no resistant $S$. typhimurium detected in their faeces.

The resistant $S$. typhimurium organisms isolated from the livers of the chicken possessed the same antibiotic-resistance pattern as those isolated from the faeces. Of the 68 chicken given ampicillin, neomycin, streptomycin or 0.05 per cent. oxytetracycline, 48 had antibiotic-resistant $S$. typhimurium in their livers. Not only was this a much higher proportion than was the case in the other chicken given antibiotics, but the cultures from the livers of all 48 appeared to consist entirely of resistant organisms. Resistant S. typhimurium organisms were isolated from only 5 of the 17 chicken given no antimicrobial agents and in 4 of these 5 chicken the resistant organisms were outnumbered by sensitive organisms. Since resistance transfer had been shown to take place between salmonellae in selenite broth (see Materials and methods), the medium that had been used for culturing the livers of the chicken, the experiment with ampicillin-containing food was repeated; in this series, the livers were cultured direct on plates of solid medium instead of being previously enriched in selenite broth. A few colonies grew on the plates seeded with material from the livers of 5 of the 17 chicken and all of them possessed the resistance pattern ASN.

The effect of feeding diets containing antimicrobial agents on transfer systems involving poor recipients or poor donors was also studied. When a diet containing 0.01 per cent. of ampicillin was given to 17 chicken given a good donor F17, with the resistance pattern A S N T, followed by the poor recipient 


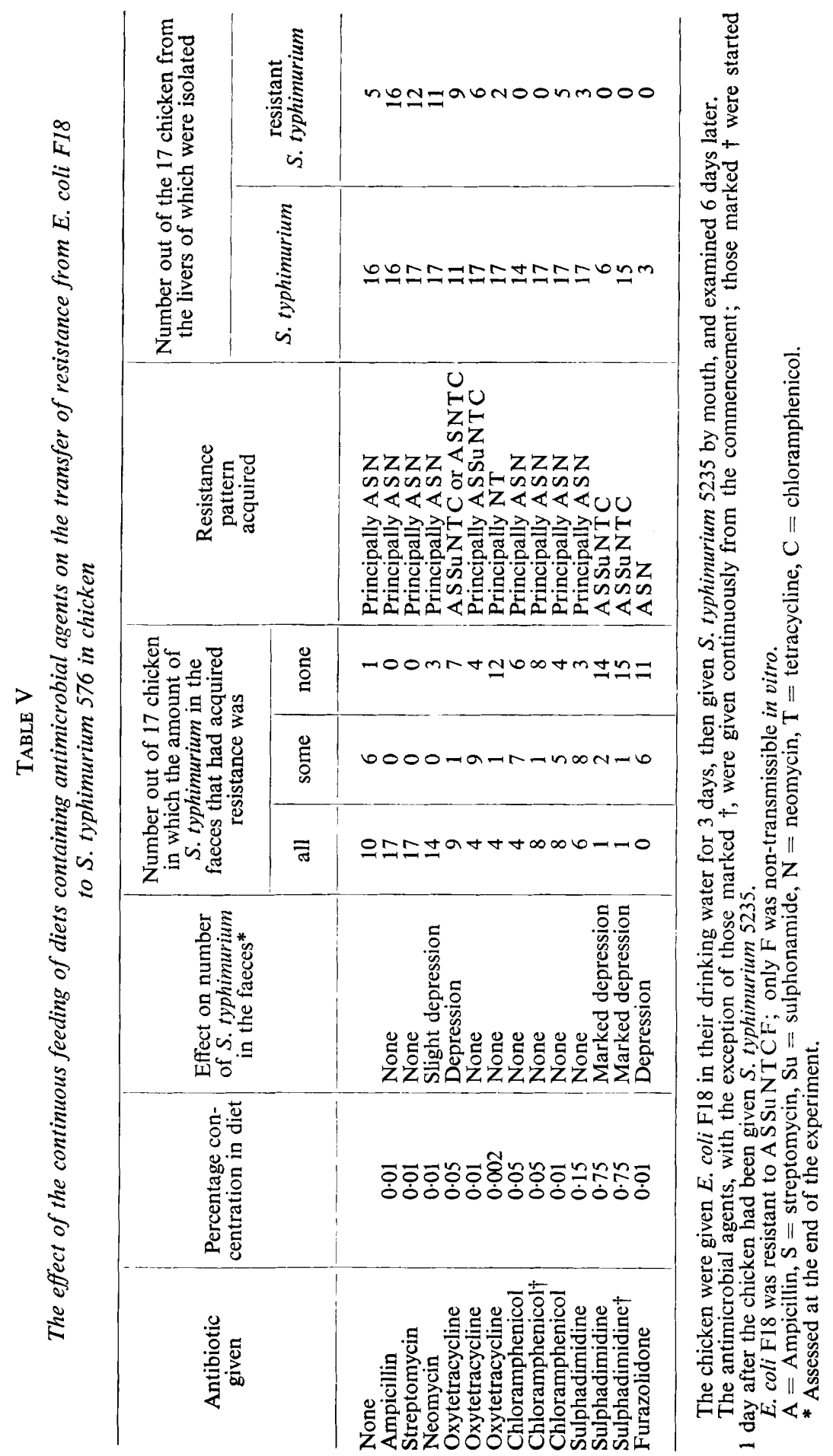


$S$. thompson, the faeces of 9 of the chicken yielded $S$. thompson with the resistance pattern $\mathrm{ASN}$; the faeces of 3 of 17 chicken in a control series given no antibiotic yielded $S$. thompson (A S N). When a similar experiment was conducted with the poor donor P113, resistance pattern S Su TC, and the good recipient $S$. typhimurium 576 , but with 0.01 per cent. streptomycin as the test antibiotic, $S$. typhimurium organisms with the same resistance pattern as that of P113 were isolated from the faeces of 6 chicken, whereas only one of the control chicken not given antibiotic yielded such an organism (table II).

The distribution of chloramphenicol in the alimentary tract after oral administration: the site in the alimentary tract in which resistance transfer occurs

Since the feeding of diets containing chloramphenicol had little effect either on the numbers of salmonellae in the faeces or on the transfer of chloramphenicol resistance, experiments were performed to determine the distribution in the alimentary tract of orally administered chloramphenicol and the site in which resistance transfer occurs.

Six 8-day-old chicken were fed ad libitum on meal containing 0.05 per cent. chloramphenicol. Three were killed 1 day later, and three 6 days later. Blobs of chyme, in approximately $0.2 \mathrm{~g}$ amounts, from the crop, the gizzard, the upper, middle and lower thirds of the small intestine, the caeca and the cloaca of each chick were placed on the surface of petri plates of nutrient agar that had been lightly seeded with a broth culture of coagulase-negative staphylococci highly sensitive to chloramphenicol. The plates were incubated at $37^{\circ} \mathrm{C}$ for $24 \mathrm{hr}$. With the samples from all 6 chicken, there were wide zones of bacterial inhibition around the chyme from the crop, narrow zones around the chyme from the gizzard and no zones at all around the chyme from the other regions of the alimentary tract. By a modification of this technique, chloramphenicol was demonstrated in the blood of the chicken.

The sites in the alimentary tract in which resistance transfer may occur were determined by giving 10 one-day-old chicken drinking water containing $E$. coli F18, transmissible resistance pattern A S Su N T C, for 3 days and then infecting them orally with the nalidixic acid-resistant mutant of S. typhimurium 5235 . Crop contents and faeces were taken from each chick $0, \frac{1}{2}, 1 \frac{1}{2}, 3,4 \frac{1}{2}, 7$ and $24 \mathrm{hr}$ later and then at 24-hr intervals, and examined in the usual way for $E$. coli and antibiotic-resistant $S$. typhimurium. Large numbers of $E$. coli F18 and $S$. typhimurium were present in the crop during the first $7 \mathrm{hr}$, but thereafter their numbers in this organ were low; none of these $S$. typhimurium organisms was found to be antibiotic-resistant. Larger numbers of $E$. coli F18 were usually found in the faeces than in the crop, but only occasionally were they the major component of the $E$. coli flora. Antibiotic-resistant $S$. typhimurium, resistance pattern $\mathrm{ASN}$, were found in the faeces of 4 of the 10 chicken, appearing first in one chick 1 day after infection with $S$. typhimurium and first in the other three at 2, 4 or 6 days after infection. Resistant $S$. typhimurium were always found in the faeces of these 4 chicken at each subsequent daily examination. The 4 chicken were killed 1-6 days after resistant $S$. typhimurium were first found in 
their faeces and the contents of the crop, gizzard, anterior and posterior small intestine, caeca and cloaca of each one examined. The findings were similar in each chick. E. coli F18 and S. typhimurium were not found in the crop, gizzard or small intestine. They were present in large numbers in the caeca and in smaller numbers in the cloaca, the S. typhimurium possessing the resistance pattern ASN.

The transfer of antibiotic-resistance between chicken infected with antibioticresistant $E$. coli and chicken infected with antibiotic-sensitive S. typhimurium

Eighteen chicken were infected with $E$. coli F18, transmissible resistance pattern ASSu N TC, and another group of 18 chicken were infected with the

\section{TABLE VI}

Transfer of resistance between two groups of 18 chicken, infected orally with E. coli F18 or a nalidixic acid-resistant mutant of $S$. typhimurium 5235, 1 day before being mixed

\begin{tabular}{|c|c|c|c|c|}
\hline \multirow{2}{*}{$\begin{array}{l}\text { Chicken infected } \\
\text { initially with }\end{array}$} & \multirow{2}{*}{$\begin{array}{l}\text { Days after } \\
\text { being mixed }\end{array}$} & \multicolumn{3}{|c|}{$\begin{array}{c}\text { Number out of } 18 \text { chicken from whose faeces were } \\
\text { isolated } S \text {. typhimurium that were }\end{array}$} \\
\hline & & all sensitive & $\begin{array}{l}\text { sensitive and } \\
\text { resistant* }\end{array}$ & all resistant* \\
\hline S. typhimurium 5235 & $\begin{array}{r}2 \\
9 \\
16 \\
23 \\
30 \\
37\end{array}$ & $\begin{array}{r}16 \\
12 \\
10 \\
11 \\
4 \\
4\end{array}$ & $\begin{array}{l}2 \\
4 \\
1 \\
1 \\
1 \\
2\end{array}$ & $\begin{array}{l}0 \\
2 \\
3 \\
3 \\
3 \\
2\end{array}$ \\
\hline E. coli F18 & $\begin{array}{r}2 \\
9 \\
16 \\
23 \\
30 \\
37\end{array}$ & $\begin{array}{l}5 \\
5 \\
7 \\
6 \\
2 \\
3\end{array}$ & $\begin{array}{l}1 \\
2 \\
2 \\
2 \\
1 \\
1\end{array}$ & $\begin{array}{l}0 \\
1 \\
3 \\
2 \\
2 \\
1\end{array}$ \\
\hline
\end{tabular}

The chicken were 1 day old when they were given $10^{9}$ viable organisms of either $E$. coli F18 or $S$. typhimurium. E. coli F18 possessed the transmissible resistance pattern AS Su NTC.

* Resistance pattern ASN.

nalidixic acid-resistant mutant of $S$. typhimurium 5235. The two groups were mixed 1 day after infection and the results of periodic examinations of their faeces are given in table VI. On the 2 nd day after mixing, $E$. coli F18 formed the major part of the $E$. coli faecal flora in most of the 18 chicken given that strain; a small number of these organisms were also found at this time in the faeces of 3 of the 18 chicken that had been given $S$. typhimurium. At 9 and 16 days, the distribution of $E$. coli F18 was similar in both groups of chicken, these organisms forming the major component of the $E$. coli faecal flora in only a few chicken in each group; they were present in small numbers in the others. Thereafter, they were either absent or present in only small numbers in all the 
chicken. Once resistant S. typhimurium was found in the faeces of any particular chick, it was usually found at subsequent examinations.

\section{Antibiotic-resistance transfer in calves}

Eight calves were given, by mouth, $E$. coli F18, transmissible resistance pattern ASSu NTC, and then the nalidixic acid-resistant mutant of S. typhimurium 5235, phage-type 29, 1 day later. Three showed no signs of ill-health thereafter and at each daily examination during the next 10 days their faeces yielded no $S$. typhimurium 5235 or only small numbers of the strain, all antibioticsensitive, were isolated.

The remaining 5 calves developed severe infections and died 2-5 days after receiving $S$. typhimurium 5235. At each daily examination the faeces of all 5 calves yielded a good growth of these organisms and so did their organs at necropsy. Only sensitive organisms of strain 5235 were found in 2 of the calves. A small proportion of the strain 5235 organisms isolated at most of the daily examinations of the faeces of the other 3 calves possessed the resistance pattern A S N. Similar organisms were also found at necropsy in the small intestine, colon, caecum, mesenteric lymph-glands, liver and spleen of each of 2 of these 3 calves; in some instances they comprised about 10 per cent. of the total population of strain 5235. Only the caecal contents and faeces were examined at the necropsy of the third calf. Both materials yielded some resistant 5235 organisms, their resistance patterns being (i) $\mathrm{ASN}$; (ii) $\mathrm{SSuC}$; or (iii) A S Su NC.

\section{Antibiotic-resistance transfer in pigs}

It was not possible to study resistance transfer from $E$. coli to $S$. typhimurium in pigs because of the inability of the two good recipient strains of S. typhimurium, 576 and 5235, to establish adequate colonisation in the alimentary tract of these animals. For example, when 4 pigs were given 1010 viable organisms of each of these strains on two different occasions by mouth, only small numbers were found in the faeces $24 \mathrm{hr}$ later, and none at all at subsequent daily examinations. For this reason, the studies in pigs were confined to the transfer of resistance from $E$. coli to $E$. coli. Most of the strains used as prospective recipients were derived from serotypes pathogenic for pigs. They colonised the alimentary tract poorly, probably because the pigs were beyond the age of susceptibility to clinical $E$. coli infection. In these studies, an $E$. coli faecal flora that was principally tetracycline-resistant was produced in pigs by feeding them for several days on a diet containing approximately 0.01 per cent. of oxytetracycline. Immediately after this diet was discontinued, the pigs were fed on ordinary food and given an oral dose of 1010 viable organisms of a prospective recipient strain of $E$. coli which was a nalidixic acid-resistant mutant. The faeces of the pigs were cultured daily for the next 10 days on MacConkey medium containing nalidixic acid and oxytetracycline.

No transfer of resistance was detected in 32 pigs given prospective recipient strains of serotypes $0141: \mathrm{K} 85 \mathrm{ab}, 88 \mathrm{ab}, 0141: \mathrm{K} 85 \mathrm{ac}$ or $0138: \mathrm{K} 81$. A few tetracycline-resistant colonies of a strain of serotype $08: \mathrm{K} 87,88 \mathrm{ab}$ were 
isolated from the faeces of 3 of 5 pigs on 2 occasions, 5-7 days after they had been given this serotype. Similar results were obtained in another 5 pigs in which the prospective recipient strain was a human pathogen, O26:K60.

More effective transfer occurred in 2 pigs in which an O139:K82 strain of $E$. coli was the prospective recipient; 1-20 tetracycline-resistant colonies of this strain were isolated daily for the first 6 days from each pig. This O139:K82 strain was a mutant resistant to streptomycin as well as to nalidixic acid; when daily doses of streptomycin $200 \mathrm{mg}$ and oxytetracycline $200 \mathrm{mg}$ were then given orally to these 2 pigs for 4 days, the numbers of tetracycline-resistant $0139: K 82$ organisms in the faeces increased and $10^{2}-10^{5}$ organisms per $\mathrm{g}$ were found at each daily examination for the next 15 days. In a similar experiment in which the $0139: K 82$ strain was given to 6 pigs that had not been fed on diets containing oxytetracycline, and in which tetracycline-resistant $E$. coli formed only a minority of the $E$. coli faecal flora, being present in numbers of $10^{2}-10^{4}$ viable organisms per $g$ of faeces, tetracycline-resistant organisms of the recipient strain were found in one pig on one occasion. However, after oxytetracycline and streptomycin treatment, tetracycline-resistant organisms of the recipient strain were isolated from the faeces of all the pigs on each of the next 14 days in numbers of $10^{3}-10^{5}$ per $\mathrm{g}$.

In the pigs in the above experiments from which tetracycline-resistant recipient organisms were isolated, the transmissible nature of this resistance was confirmed by transfer in vitro to $E$. coli $\mathrm{K} 12 \mathrm{~F}^{-}$.

\section{DiscussION}

The results indicate that transfer of antibiotic resistance can take place at a high rate in chicken with a normal alimentary flora and in the absence of antibiotics. However, it is clear that the demonstration of resistance transfer between two strains in vitro does not necessarily imply that transfer will also occur in vivo. This was far from the case. To obtain a significant degree of transfer in vivo in the absence of antibiotic, it was essential to use a pair of strains in which the passage of $\mathrm{R}$ factors from one to the other occurred at a high rate in vitro. It was also important that both strains should be reasonably good colonisers of the alimentary tract. These conditions in regard to in-vitro transmissibility and colonisation were satisfied by several pairs of strains, the donors being one or other of several strains of $E$. coli and the recipients one or other of three strains of $S$. typhimurium, those of phage-types 29 and 36 being outstanding recipients in vivo. It is of interest in relation to colonising ability that two good donors in chicken, E. coli P18 and P233, were isolated originally from pigs. Colonisation in chicken implies persistence in reasonably high numbers in the caeca, because it is here that resistance transfer was found to occur. The importance of colonising ability is well illustrated by the studies in which chicken were mixed together after receiving either the prospective donor strain, E. coli F18, or the prospective recipient strain, S. typhimurium 5235. The spread of $\mathrm{R}$ factors stopped when there was a sharp decline in the numbers of F18 in the alimentary tracts of the chicken.

The present studies illustrate the dangers of generalisations regarding the 
significance of in-vivo transfer of $\mathrm{R}$ factors. For example, the test strains differed greatly in their recipient ability. With the $S$. typhimurium strains, extreme differences were evident between strains of the same phage-type. There were also great differences in donor ability between strains with the same transmissible resistance pattern, e.g., E. coli $\mathrm{H} 44$ and F18 of pattern A S Su N T C, and F20 and F21 of pattern T. It is noteworthy that 45 of the 50 strains of $E$. coli that were resistant to tetracycline only, resembled F20 in transmitting $\mathrm{T}$ in vitro at a low rate. If strains of this kind had been the only ones examined, it would have seemed reasonable to conclude that $\mathrm{R}$-factor donation from strains with the pattern $T$ is of little more than academic interest, at least in an environment devoid of antibiotic; the in-vivo results obtained with the effectively transmitting strain, F21, and to a lesser extent with F22, indicate that this would have been wrong.

The effect of oral antibiotic administration on in-vivo transfer varied. Chloramphenicol appeared to have no influence at all, probably because none of it reaches the caeca, the organs in which resistance transfer was found to occur. Usually, antibiotic administration increased the rate of in-vivo transfer. This was noticeably so with the system in which the donor was E. coli F18 and the recipient was a phage-type $29 S$. typhimurium strain, 5235; here transfer occurred at a fairly high rate even in the absence of antibiotics. This finding and the observation that resistance transfer between these strains also occurs in calves, accords with the observations of Anderson (1965) and Anderson and Lewis (1965) that a great increase has occurred in recent years in the incidence of R-factor-bearing strains of S. typhimurium phage-type 29 in calves in Britain, an increase that has coincided with the rapid development of the intensive method of calf-rearing in this country. As these authors observed, this system is not only conducive to the spread of salmonella organisms amongst the calf population but is one in which many different antibiotics are used on a large scale.

\section{SUMMARY}

In mixed culture experiments, great variation was found between different strains of Escherichia coli and salmonellae in their ability to transmit or to receive antibiotic resistance ( $\mathrm{R}$ factors).

Resistance transmission was demonstrated to occur in the alimentary tract of chicken given strains by mouth, but only between strains that were known to be good donors and recipients in vitro and able to colonise the alimentary tract reasonably well. Sometimes, the $\mathbf{R}$ factors appeared to infect the recipient organisms in the alimentary tract in epidemic form. Some strains of S. typhimurium, including one of phage-type 29 , were outstandingly good recipients in vivo; organisms of these strains that had received $\mathrm{R}$ factors were not uncommonly found in the liver as well as in the faeces of experimental chicken. It was not necessary to give each chick both the prospective donor and the prospective recipient strain. For example, transfer occurred in chicken that were mixed together after some had been given a prospective donor strain of $E$. coli and others a prospective recipient strain of $S$. typhimurium. 
During transfer studies in chicken, feeding with a diet containing an antibiotic that featured in the transmissible resistance pattern of the prospective donor strain occasionally had a suppressive effect on the numbers of prospective recipient organisms in the faeces. Usually, however, it led to a great increase in the proportion of R-factor-containing recipient organisms, both in the faeces and in the liver. Chloramphenicol did not appear to influence the situation. This was because the drug is absorbed in the anterior part of the alimentary tract and well before the caeca, the organs in which resistance transfer was found to occur.

Resistance transfer from $E$. coli to $S$. typhimurium was also demonstrated to occur in the alimentary tract of calves but not of pigs. Poor colonisation of the alimentary tract by $S$. typhimurium was the probable reason for the failure in pigs; transfer from $E$. coli to $E$. coli, however, was demonstrated in these animals.

The results are discussed with particular reference to the high incidence of antibiotic-resistant strains of S. typhimurium in intensively reared calves.

I am grateful to Miss Diane Poulton and Miss Carole Smith for their capable technical help. My thanks are also due to Mr L. J. Abbott, Dr E. S. Anderson, Mrs Esther Johnson, Dr K. C. Sellers, Mr W. J. Sojka and Dr Joan Taylor for advice and assistance in various ways. All the chicken used in this work were a gift from Arbor Acres (U.K.) Ltd.

\section{REFERENCES}

ANDERSON, E. S. . $\quad$. $\quad$. $\quad$. $\quad$. 1965. Br. Med. J., 2, 1289.

Anderson, E. S., AND Lewis, M. J. $\quad$. 1965. Nature, Lond., 206, 579.

GuINÉE, P. A. M. . . . . . 1965. Antonie van Leeuwenhoek, 31, 314.

KASUYA, M. . . . . . 1964. J. Bact., 88, 322.

Miles, A. A., Misra, S. S., AND Irwin, 1938. J. Hyg., Camb., 38, 732. J. 0 .

Salzman, T., and Klemm, L. ～～～～～1968. Proc. Soc. Exp. Biol. Med., 128, 392.

SMITH, H. W.

WALtON, J. R. - $\quad$. $\quad$. $\quad$. $\quad$. 1966. Nature, Lond., 211, 312

Watanabe, T. . . . . . . 1963. Bact. Rev., $27,87$. 